\title{
Ketoprofen/ethyl Cellulose Nanofibers Fabricated Using an Epoxy-coated Spinneret
}

\author{
Xiao-Yan Li, Deng-Guang Yu", Cai-Tao Fu, Rui Wang, Xia Wang* \\ School of Materials Science \& Engineering, University of Shanghai for Science and Technology, Shanghai, China \\ Email: *ydg017@usst.edu.cn, wwangxia@usst.edu.cn \\ Received May, 2013
}

\begin{abstract}
The present study investigates the preparation of sustained release drug-loaded nanofibers using a novel epoxy-coated spinneret. With ethyl cellulose (EC) and ketoprofen (KET) as the filament-forming matrix and the active pharmaceutical ingredient, Drug-loaded composite nanofibers are generated smoothly and continuously with few user interventions. Field-emission scanning electron microscopic observations demonstrated that the composite nanofibers prepared using the epoxy-coated spinneret have better quality than those from a traditional stainless steel spinneret in terms of diameter and its distribution. Both of the composite nanofibers are in essential a molecular solid dispersion of EC and KET based on the hydrogen bonding between them, as verified by XRD and ATR-FTIR results. In vitro dissolution tests show that the nanofibers resulted from the new spinneret provide a finer sustained KET release profile than their counter-parts. Epoxy-coated spinneret is a useful tool to facilitate the electrospinning process through the prevention of clogging for generating high quality nanofibers.
\end{abstract}

Keywords: Spinneret; Electrospinning; Nanofibers; Sustained Drug Release; Ethyl Cellulose

\section{Introduction}

Electrospinning has attracted increased attentions as a "top-down" nanotechnology for nanofiber production owing to its simplicity and cost effectiveness [1,2]. Significant progress has been made in this process throughout the past few years and electrospinning has advanced its applications in many fields, including pharmaceutics. Electrospun nanofibers show great promise for developing many types of novel drug delivery systems (DDS) due to their unique properties $[3,4]$. However, one of the common problems associated with the preparation of drug-loaded nanofibers is the frequent clogging of the spinneret during the electrospinning process, and by which often the quality of resulted nanofibers are deteriorated, especially when a high-volatility solvent is used to prepare a polymer solution $[5,6]$.

Most recently, a modified coaxial electrospinning process, in which only organic solvents were exploited as sheath fluids, is reported to successfully prevent the clogging during the electrospinning process. The organic solvents as sheath fluids can stabilize the electrospinning process and assists the generation of high quality nanofibers (in terms of their size distribution, uniformity and surface morphology) [7-9]. Although the modified coaxial process opens a new route to generate nanofibers from poly-

*Corresponding author. mer solutions through partially replacing the traditional interface between polymer jets and the atmosphere by one between polymer jets and sheath solvents, the introducetion of additional fluids would increase the complexity of the electrospinning system and make the implementation of electrospinning more difficult than a traditional single fluid electrospinning.

Typically an electrospinning system comprises of four major components: a high-voltage power supply, a collector, a fluid driving device and a spinneret. Some modifications to the system involve the use of alternative collectors (such as rotating drum, plate collector with guiding electrodes, functionalized target electrodes and moveable collector) or the use of auxiliary apparatus to facilitate the electrospinning process, for example at raised temperatures or increased humidity [7]. However, more significant modifications to electrospinning concern the spinneret, by which different processes have been successfully developed, such as coaxial electrospinning, side-by-side and needleless electrospinning [10-12]. Thus new strategies for improving the electrospinning process can potentially depend on the innovation of spinneret.

In the present study, a new spinneret, characterized by an epoxy resin (EP) coating on the nozzle of a stainless steel capillary, was developed for the continuous preparation of ketoprofen (KET)-loaded ethyl cellulose (EC) 
nanofibers. KET, a non-steroidal anti-inflammatory drug that has poor water solubility, was exploited as the model drug. EC is a derivative of cellulose in which a defined percentage of the hydroxyl groups of the repeating glucose units are substituted with ethyl ether groups. It fulfills all the requirements of major pharmacopoeias (USP, EP, JP) and food regulations. EC is an inert, hydrophobic polymer and is essentially tasteless, odourless, colorless, non-caloric, and physiologically inert. It has long been used as solventbased tablet and pellet coating, tablet binder, to prepare microcapsules and microspheres, and both as film- and matrix- forming material for sustained-release dosage forms [13]. Most recently, EC was selected as the drug carrier and polymer matrix to generate composite fibers and microparticles to achieve sustained-release profiles $[13,14]$.

\section{Experimental}

\subsection{Materials}

KET was purchased from Wuhan Fortuna Chemical Co. Ltd. (Hubei, China). EC (6 $\mathrm{mPa} \cdot \mathrm{s}$ to $9 \mathrm{mPa} \cdot \mathrm{s})$ was obtained from Aladdin Chemistry Co., Ltd (Shanghai, China). Anhydrous ethanol was purchased from Sino-pharm Chemical Reagent Co., Ltd. (Shanghai, China). Epoxy (EP) resin and its hardener were purchased from the Jiaojiang Qinfen Chemical Factory (Taizhou, Zhejiang, China). All other chemicals used were analytical grade, and water was doubly distilled before use.

\subsection{Electrospinning}

The electrospinnable solutions were prepared by dissolveing $24 \mathrm{~g} \mathrm{EC}$ and $3 \mathrm{~g} \mathrm{KET}$ in $100 \mathrm{~mL}$ ethanol. A syringe pumps (KDS200, Cole-Parmer, IL, USA) and a highvoltage power supply (ZGF $60 \mathrm{kV} / 2 \mathrm{~mA}$, Shanghai Sute Corp., Shanghai, China) were used in the experiments. All electrospinning processes were carried out under ambient conditions $\left(21^{\circ} \mathrm{C} \pm 2{ }^{\circ} \mathrm{C}\right.$ with a relative humidity $64 \% \pm 6 \%$ ). A traditional stainless steel capillary and a homemade epoxy-coated spinneret and were used to conduct the electrospinning processes, and their nanofibers are termed as F1 and F2, respectively. The electrospinning process was recorded using a digital video recorder (PowerShot A490, Canon, Tokyo, Japan). For optimization, the applied voltage was fixed at $15 \mathrm{kV}$, and the fibers were collected on an aluminum foil at a distance of $20 \mathrm{~cm}$. A DSA100 drop shape analysis instrument (Krüss GmbH, Hamburg, Germany) were used to explore the interfacial tensions between the polymer solutions and nozzles of the spinnerets.

\subsection{Characterization}

\subsubsection{Morphology}

The morphology of the fiber mats was assessed using an
S-4800 field emission scanning electron microscope (FESEM, Hitachi, Tokyo, Japan). Prior to the examination, the samples were platinum sputter-coated under a nitrogen atmosphere to render them electrically conductive. Images were recorded at an excitation voltage of 10 $\mathrm{kV}$. The average fiber diameter was determined by measuring their diameters in FESEM images at more than 100 places using the NIH Image $J$ software (National Institutes of Health, MD, USA).

\subsubsection{Physical Status and Compatibility}

The X-ray diffraction analysis (XRD) was conducted using a D/Max-BR diffractometer (RigaKu, Japan) with $\mathrm{Cu} \mathrm{K} \alpha$ radiation in a $2 \theta$ range of $5^{\circ}$ to $60^{\circ}$ at $40 \mathrm{mV}$ and $300 \mathrm{~mA}$. Attenuated total reflectance-Fourier transform infrared (ATR-FTIR) spectroscopy was carried out on a Nicolet-Nexus 670 FTIR spectrometer (Nicolet Instrument Corporation, Madison, USA) at a range of $500 \mathrm{~cm}^{-1}$ to $4000 \mathrm{~cm}^{-1}$ and a resolution of $2 \mathrm{~cm}^{-1}$.

\subsubsection{In Vitro Dissolution Tests}

In vitro dissolution tests were carried out according to the Chinese Pharmacopoeia (2005 ed.) Method II, which is a paddle method using a RCZ-8A dissolution apparatus (Tianjin University Radio Factory, Tianjin, China) was used. Drug-loaded nanofibers $(200 \mathrm{mg})$ were placed in $600 \mathrm{~mL}$ physiological saline (PS, $0.9 \mathrm{wt} \%$ ) at $37^{\circ} \mathrm{C} \pm 1$ ${ }^{\circ} \mathrm{C}$. The instrument was set to $50 \mathrm{rpm}$, providing sink conditions with $C<0.2 C_{\mathrm{s}}$. At predetermined time points, $5.0 \mathrm{~mL}$ aliquots of the samples were withdrawn from the dissolution medium and replaced with fresh medium to maintain a constant volume. After filtration through a $0.22 \mu \mathrm{m}$ membrane (Millipore, MA, USA) and appropriate dilution with PS, the samples were analyzed at 260 $\mathrm{nm}$ using a UV-vis spectrophotometer (UV-2102PC, Unico Instrument Co. Ltd., Shanghai, China). The concentration of released KET was back calculated from the data obtained against a predetermined calibration curve. The experiments were carried out six times, and the accumulative percent reported as mean values was plotted as a function of time $(T, \mathrm{~h})$.

\section{Results and Discussion}

A traditional spinneret used in this study was a standard 20G metal needle (with an inner and outer diameter of 0.60 and $0.91 \mathrm{~mm}$, respectively). This is made of $06 \mathrm{Cr}$ 19Ni10 (GB24511 in China) austenitic stainless steel, comprising steel, $\mathrm{C}(\leq 0.07 \%), \mathrm{Cr}(17.00 \%-19.00 \%), \mathrm{Ni}$ $(8.00 \%-10.00 \%), \mathrm{Mn}(\leq 2.00 \%), \mathrm{Si}(\leq 1.00 \%)$ and traces of $\mathrm{S}$ and P. To coat the needle in EP resin, EP and the hardener were mixed and then the resultant mixture was rapidly applied around the needle tip, taking care not to obstruct the aperture, the design method and a digital picture of the EP-coated spinneret are shown in Figures 
1(a) and (b).

A digital image of the arrangement of apparatus for conducting the electrospinning process is shown in Figures 2(a) and (b). A syringe pump was used to drive the fluid and an alligator clip to connect the spinneret (the non-coated part of the Teflon-coated spinneret) to the high voltage power supply. A typical fluid jet traveling process is illustrated in Figures 2(c) and (d). Typically, a straight thinning jet is emitted from the Taylor cone, and is then followed by a bending and whipping instability region with loops of increasing size. Few clogging was observed in this process, which ran smoothly and continuously with minimum user intervention. In sharp contrast, electrospinning using a traditional metal spinneret was clogged from time to time (the inset of Figure 2(b)).

An experiment was performed to determine the contact angles of the co-dissolving solution on a EP-coated steel surface and a stainless steel plate. The results are shown in Figure 3. A contact angle of $94 \pm 6^{\circ}$ is observed on a EP-coated plate, significantly larger than the $41 \pm 4^{\circ}$ seen on the steel plate $(n>10)$. This indicates that the interfacial tension between the EP-coated surface and co-dissolving solution was smaller than that between the solution and steel, and thus should do favor to the electrospinning process.

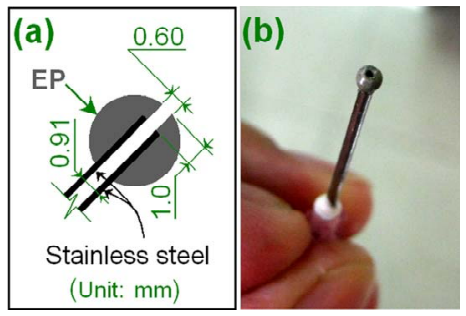

Figure 1. The design of an epoxy-coated spinneret head (a) and its digital photo (b).

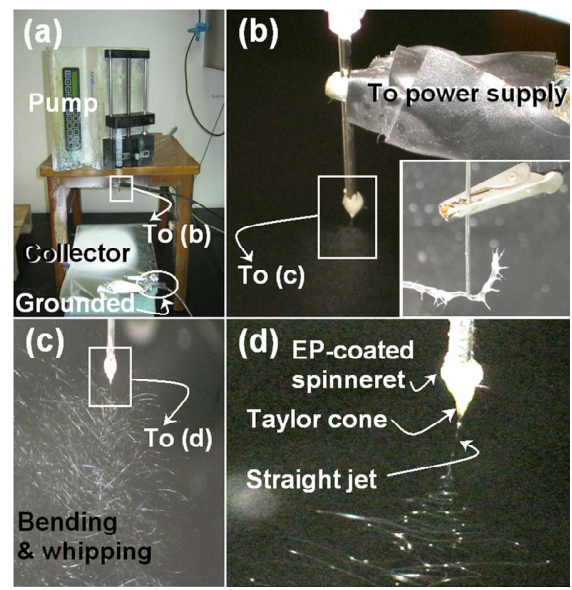

Figure 2. The arrangement of the apparatus (a and $b$ ) and observations of the electrospinning process (c and $d$ ). The inset in b shows a typical clogging in an electrospinning process using a metal spinneret.
As shown in Figure 4, Nanofibers F1 and F2 had linear structures without distinct beads-on-a-string morphology. No drug particles appeared on the surface of the fibers, indicating good compatibility between EC and KET. The nanofibers F1 prepared through the metal spinneret had average diameters of $820 \mathrm{~nm} \pm 260 \mathrm{~nm}$ (Figure 4(a)). The nanofibers F2 prepared through the EP-coated spinneret had and average diameters of $690 \mathrm{~nm} \pm 150 \mathrm{~nm}$ (Figure 4(b)). These results demonstrated that EP-coated spinneret could improve the generated nanofibers' quality with smaller diameter and narrower diameter's distribution, and also that the electrical energy might exert more efficacious drawing on the spinning solutions when the EP-coated spinneret is exploited.

XRD tests were conducted to determine the physical status of KET in the nanofibers (Figure 5). Numerous distinct reflections were found in the XRD pattern of pure KET, demonstrating that the pure drug is a crystalline material. The diffraction patterns of EC showed a diffuse background pattern with two diffraction halos, indicating that the polymer is amorphous. In the patterns of nanofibers F1 and F2, no characteristic reflections of KET were found. This observation indicates that KET

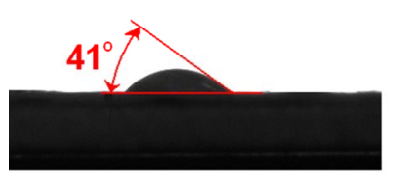

Stainless steel surface

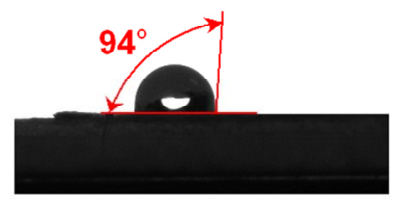

Epoxy-coated surface
Figure 3. Experiments to investigate the influence of spinneret composition on the electrospinning process, the contact angle of a spinnable solution on a stainless steel plate and on an EP-coated plate.

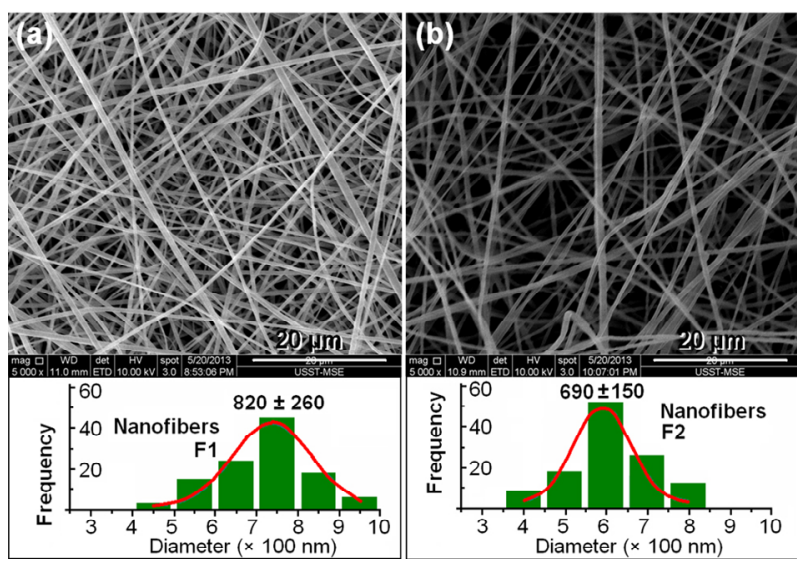

Figure 4. FESEM images of the nanofibers from different spinneret and their diameters' distributions: (a) an EP-coated spinneret, (b)a traditional stainless steel capillary. 
was no longer present as a crystalline material but was converted into an amorphous state.

Compatibility among the components, which was investigated through FTIR analysis (Figure 6), is essential for producing high-quality and stable nanofibers. Second-order interactions such as hydrogen bonding, electrostatic interactions, and hydrophobic interactions improve compatibility. KET and EC molecules possess free hydroxyl (acting as potential proton donors for hydrogen bonding) and carbonyl (potential proton receptors) groups. Therefore, hydrogen-bonding interactions may occur within the KET-loaded EC nanofibers. Two welldefined sharp peaks at 1698 and $1657 \mathrm{~cm}^{-1}$ were observed for pure crystalline KET. The former was assigned to the stretching vibration of the carbonyl group in the KET dimer, whereas the latter was assigned to the stretching of the ketone group. The peak at $1698 \mathrm{~cm}^{-1}$ was observed because KET molecules in crystalline form are bound together in dimers. However, the peak at $1698 \mathrm{~cm}^{-1}$ disappeared in the spectra of F1 and F2, indicating the breakage of the KET dimers and the formation of hydrogen bonds between the EC hydroxyl group and the KET carbonyl group. By interacting with the polymer, KET molecules are less likely to form the dimers that are essential for the formation of a crystal lattice [3].

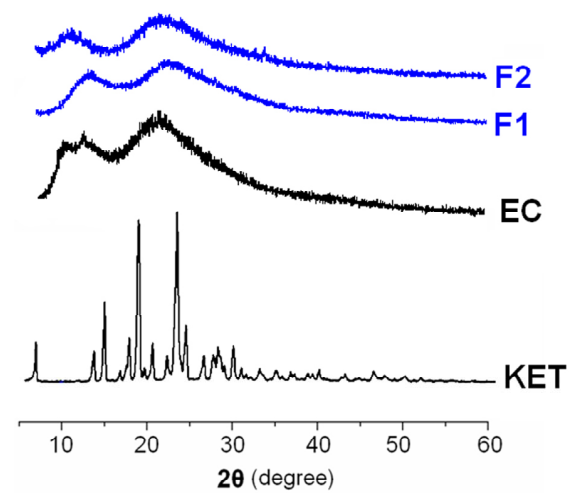

Figure 5. X-ray diffraction patterns.

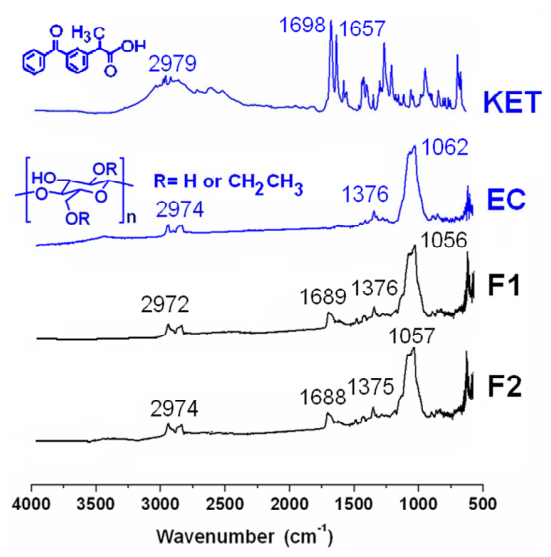

Figure 6. ATR-FTIR spectra.
The in vitro drug release profiles of the two nanofibers are shown in Figure 7. As expected, the nanofibers of F1 and $\mathrm{F} 2$ could provide fine sustained drug release profiles. The KET release profiles from the KET-loaded EC nanofibers were analyzed using the Peppas equation [15]

$$
Q=\mathrm{k} t^{\mathrm{n}}
$$

where $Q$ is the drug release percentage, $t$ is the release time, $\mathrm{k}$ is a constant reflecting the structural and geometric characteristics of the fibers, and $\mathrm{n}$ is the release exponent that indicates the drug release mechanism.

The regressed result for the nanofibers of F1 and F2 are $Q_{1}=18.7 t_{l}^{0.40}\left(R_{l}^{2}=0.9908\right)$, and $Q_{2}=21.6 t_{2}^{0.37}\left(R_{l}^{2}\right.$ $=0.9921)$, indicating that the drug release from the composite nanofibers was controlled via a typical Fickian diffusion mechanism by a value of the release exponent 0.40 and 0.37 (less than 0.45 ). The results also suggested that nanofibers F2 provided a better sustained release profiles than nanofibers F1 in terms of the accumulative release content after $24 \mathrm{~h}$ dissolution tests, which should be attributed to the more even diameter distributions of nanofibers F2.

\section{Conclusions}

A new EP-coated spinneret was developed and was exploited to conduct electrospinning for generating drugloaded nanofibers that could provide sustained drug release profiles with EC as the polymer matrix. The electrospinning process could run smoothly and continuously with few user interventions on the nozzle clogging of spinneret. FESEM observations demonstrated that the composite nanofibers prepared using the epoxy-coated spinneret have better quality than those from a traditional stainless steel spinneret in terms of diameter and its distribution. Both the composite nanofibers were in essential a composite of EC and KET based on the hydrogen bonding between them, as verified by DSC and ATRFTIR results. In vitro dissolution tests showed that the nanofibers resulted from the new spinneret provided a better sustained KET release profile than their counterparts. Epoxy-coated spinneret is a useful tool to facilitate

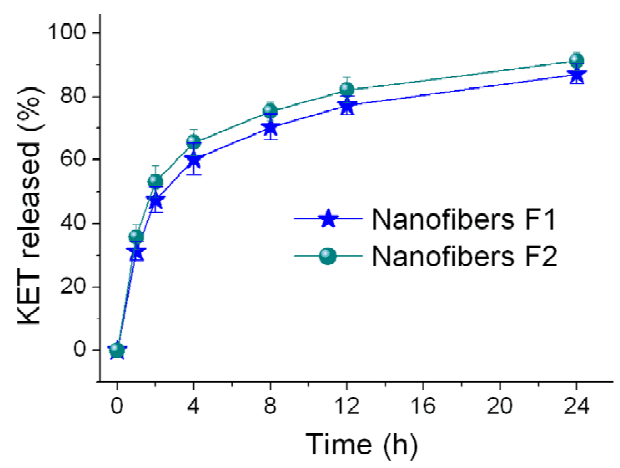

Figure 7. The in vitro dissolution tests $(n=6)$. 
the electrospinning process through prevention of clogging for generating high quality nanofibers.

\section{Acknowledgements}

This work was supported by the Natural Science Foundation of Shanghai (No.13ZR1428900), the Key project of Shanghai Municipal Education Commission (No.13ZZ113), the Innovation project of University of Shanghai for Science and Technology (No. 13XGM01), the innovation project of college student fund committee (Nos. XJ2013274 and SH201210252153).

\section{REFERENCES}

[1] D. G. Yu, L. M. Zhu, K. White and C. Branford-White, "Electrospun Nanofiber-based Drug Delivery Systems," Health, Vol. 1, No. 2, 2009, pp. 67-75. doi:10.4236/health.2009.12012

[2] A. K. Moghe and B. S. Gupta, "Co - axial Electrospinning for Nanofiber Structures: Preparation and Applications," Polymer Review, Vol. 48, No. 2, 2008, pp. 353 377. doi:10.1080/15583720802022257

[3] D. G. Yu, J. H. Yu, L. Chen, G. R. Williams and X. Wang, "Modified Coaxial Electrospinning for the Preparation of High-quality Ketoprofen-loaded Cellulose Acetate Nanofibers," Carbohydrate Polymers, Vol. 90, No. 2, 2102, pp.1016-1023. doi: 10.1016/j.carbpol.2012.06.036

[4] D.G. Yu, X. Wang, X. Y. Li, W. Chian, Y. Li and Y. Z. Liao, "Electrospun Biphasic Drug Release Polyvinylpyrrolidone/ ethyl Cellulose Core/sheath Nanofibers," Acta Biomaterialia, Vol. 9, No. 3, 2013, pp. 5665-5672. doi: 10.1016/i.actbio.2012.10.021

[5] D. G. Yu, X. Y. Li, X. Wang, W. Chian, Y. Z. Liao and Y. Li, "Zero-order Drug Release Cellulose Acetate Nanofibers Prepared Using Coaxial Electrospinning," Cellulose, Vol. 20, No. 1, 2013, pp. 379-389. doi: 10.1007/s10570-012-9824-Z

[6] D. G. Yu, W. Chian, X. Wang, X. Y. Li, Y. Li and Y. Z. Liao, "Linear Drug Release Membrane Prepared by a Modified Coaxial Electrospinning Process," Journal of Membrane Sciences, Vol. 428, No. 3, 2013, pp. 150-156. doi: 10.1016/j.memsci.2012.09.062
[7] D. G. Yu, C. Branford-White, S. W. A. Bligh, K. White, N. P. Chatterton and L. M. Zhu, "Improving Polymer Nanofiber Quality Using a Modified Co-axial Electrospinning Process," Macromolecular Rapid Communications, Vol. 32, No. 9-10, 2011, pp. 744-750. doi: 10.1002/marc.201100049

[8] D. G. Yu, L. M. Zhu, C. Branford-White, S. W. A. Bligh and K. White, "Coaxial Electrospinning with Organic Solvent for Controlling the Self-assembled Nanoparticle size," Chemical Communications, Vol. 47, No. 4, 2011, pp. 1216-1218. doi: 10.1039/c0cc03521a

[9] D. G. Yu, C. Branford-White, N. P. Chatterton, K. White, L. M. Zhu, X. X. Shen and W. Nie, "Electrospinning of Concentrated Polymer Solutions," Macromolecules, Vol. 43, No. 24, 2010, pp. 10743-10746. doi: $10.1021 / \mathrm{ma1} 024363$

[10] Z. Liu, D. D. Sun and P. Guo, "An Efficient Bicomponent $\mathrm{TiO}_{2} / \mathrm{SnO}_{2}$ Nanofiber Photocatalyst Fabricated by Electrospinning with a Side-by-side Dual Spinneret Method," Nano Letters, Vol. 7, No. 3, 2007, pp. 1081-1085. doi: 10.1021/n1061898e

[11] T. Lin, H. Wang and X. Wang, "Self-Crimping bicomponent nanofibers electrospun from polyacrylonitrile and elastomeric polyurethane," Advanced Materials, Vol.17, No.7, 2005, pp. 2699-2703. doi: $10.1002 / \mathrm{adma}$

[12] M. C. George and P. V. Braun, "Multicompartmental Materials by Electrohydrodynamic Cojetting," Angewandte Chemie International Edition, Vol. 48, No. 8, 2009, pp. 8606- 8609. doi: 10.1002/anie.200904089

[13] L. Y. Huang, D. G. Yu, C. Branford-White and L. M. Zhu, "Sustained Release of Ethyl Cellulose Micro-particulate Drug Delivery Systems Prepared Using Electrospraying," Journal of Materials Science, Vol.47, No. 3, 2012, pp. 1372-1377. doi: 10.1007/s10853-011-5913-X

[14] L. Y. Huang, C. Branford-White, X. X. Shen, D. G. Yu and L. M. Zhu, "Time-engineeringed Biphasic Drug Release by Electrospun Nanofiber Meshes," International Journal of Pharmaceutics, Vol. 436, No. 1-2, 2012, pp. 88-96. doi: 10.1016/j.ijpharm.2012.06.058

[15] N. A. Peppas, "Analysis of Fickian and Non-Fickian Drug Release from Polymers," Pharmaceutica Acta Helvetiae, Vol. 60, No. 1, 1985, pp. 110-111. 